Rapid Reviews COVID-19

\title{
Review 1: "Reopening universities during the COVID-19 pandemic: A testing strategy to minimize active cases and delay outbreaks"
}

Dominique Gibert ${ }^{1}$

${ }^{1}$ Lyon 1 University

Published on: Sep 02, 2020

DOI: $10.1162 / 2 \mathrm{e} 3983 f 5 . a a 72824 \mathrm{~d}$

License: Creative Commons Attribution 4.0 International License (CC-BY 4.0). 


\section{RR:C19 Evidence Scale rating by reviewer:}

- Potentially informative. The main claims made are not strongly justified by the methods and data, but may yield some insight. The results and conclusions of the study may resemble those from the hypothetical ideal study, but there is substantial room for doubt. Decision-makers should consider this evidence only with a thorough understanding of its weaknesses, alongside other evidence and theory. Decisionmakers should not consider this actionable, unless the weaknesses are clearly understood and there is other theory and evidence to further support it.

$* * * * * * * * * * * * * * * * * * * * * * * * * * * * * * * * * * * * * * * * *$

\section{Review:}

The paper addresses the important topic of how to manage post-confinement in order to control the epidemic spread. The case study concerns the population of a University campus counting 25000 persons. The authors use a SIR numerical model to simulate the spread of SARS-Cov2 among the student population.

The authors should give more details about the numerical method used in their study. They cite ref [7] which seems to indicate that the model is based on integration of a system of differential equations. It is our experience that, for a small population as for a University campus, stochastic models are particularly suitable to account for many type of constrains difficult to implement with the integral approach.

Some more details should be given concerning the infectious period (is it assumed to have a uniform probability?). The authors consider a duration of 3 days for this period, which seems quite short when compared with other published figures. I believe that accounting for the time delays of testing and isolating infected persons is an important issue, as we observed in the models we made for Guadeloupe. Did the authors make some sensitivity tests about such delays?

An important concern I have about this study is that the authors do not consider the University staff (teachers and others) which is also exposed to contamination. Also, it is implicitly assumed that the campus population constitutes a closed system with no interaction with outer populations. This seems a quite unrealistic assumption with 
respect to the many social interactions generally observed among students and others (for instance, many students work outside to earn money). 